\title{
Correction: Cytochrome P450 1 B1 inhibition suppresses tumorigenicity of prostate cancer via caspase-1 activation
}

\author{
Inik Chang ${ }^{1, *}$, Yozo Mitsui ${ }^{2,3, *}$, Seul Ki Kim ${ }^{1,4}$, Ji Su Sun ${ }^{1,4}$, Hye Sook Jeon ${ }^{1}$, Jung \\ Yun Kang ${ }^{1,4}$, Nam Ju Kang ${ }^{1,4}$, Shinichiro Fukuhara ${ }^{2,3}$, Ankurpreet Gill2, Varahram \\ Shahryari' ${ }^{2}$ Z. Laura Tabatabai ${ }^{5}$, Kirsten L. Greene ${ }^{5}$, Rajvir Dahiya ${ }^{2,3}$, Dong Min \\ Shin $^{1,4}$ and Yuichiro Tanaka ${ }^{2,3}$ \\ 1 Department of Oral Biology, Yonsei University College of Dentistry, Seoul, South Korea \\ 2 Department of Surgery and Division of Urology, Veterans Affairs Medical Center, San Francisco, California, United States \\ of America \\ ${ }^{3}$ Department of Urology, University of California, San Francisco, California, United States of America \\ ${ }^{4}$ BK21 PLUS Project, Yonsei University College of Dentistry, Seoul, South Korea \\ ${ }^{5}$ Department of Pathology, Veterans Affairs Medical Center and University of California, San Francisco, California, United \\ States of America \\ * These authors have contributed equally to this work \\ Published: September 25, 2018
}

Copyright: Chang et al. This is an open-access article distributed under the terms of the Creative Commons Attribution License 3.0 (CC BY 3.0), which permits unrestricted use, distribution, and reproduction in any medium, provided the original author and source are credited.

This article has been corrected: The image for \#4-3 in Figure $2 \mathrm{C}$ is incorrect. It is a duplicate image of panel \#4-2. The corrected Figure $2 \mathrm{C}$ is shown below. The authors declare that these corrections do not change the results or conclusions of this paper.

Fig $2 \mathrm{C} \# 4-2$
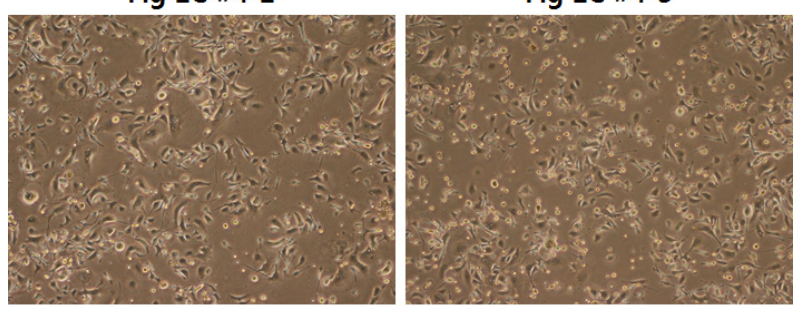

C

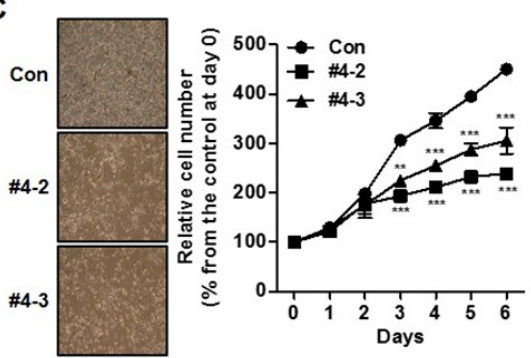

Figure 2: CYP1B1 inhibition suppresses in vitro tumorigenicity. (A and B) Expression of CYP1B1 mRNA (A) and protein (B) in CYP1B1 shRNA or control shRNA expressing PC-3 cells. Levels were determined by qRT-PCR and Western blot, respectively. (C-H) Effect of CYP1B1 knockdown on in vitro tumorigenicity. Cell proliferation as determined by MTS assay at the indicated times. Representative images of cell morphology (left panel) and quantification of cell proliferation (right panel) are shown (C). Colony formation as determined by crystal violet staining. Representative image of colonies (left panel) and quantification of stained colonies (right panel) are shown (D). Apoptotic cell death as determined by flow cytometric analysis using double staining with Annexin V-FITC and 7-AAD. Representative biparametric histograms exhibiting cell (left panel) and quantification of apoptotic cells (right panel) are shown (E). Cell cycle progression as determined by DAPI staining $(\mathrm{F})$. Cell migration $(\mathrm{G})$ and invasion $(\mathrm{H})$ capability as determined by transwell migration and invasion assay, respectively. Representative images (left panel) and quantification of assay (right panel) are shown. ${ }^{* *} p<0.01 ;{ }^{* * *} p<0.001$.

Original article: Oncotarget. 2017; 8:39087-39100. https://doi.org/10.18632/oncotarget.16598 